\title{
Fano-type interpretation of red shifts and red tails in hole array transmission spectra
}

\author{
C. Genet, M.P. van Exter, and J.P. Woerdman \\ Leiden University, Huygens Laboratory, P.O.Box 9504, \\ 2300 RA Leiden, The Netherlands \\ Optics Communications 225, 331 (2003)
}

\begin{abstract}
We present a unifying point of view which allows to understand spectral features reported in recent experiments with two-dimensional arrays of subwavelength holes in metal films. We develop a Fano analysis of the related scattering problem by distinguishing two interfering contributions to the transmission process, namely a non-resonant contribution (direct scattering) and a resonant contribution (surface plasmon excitation). The introduction of a coupling strength between these two contributions naturally induces resonance shifts and asymmetry of profiles which satisfy simple scaling relations. We also report an experiment to confirm this analysis.

PACS numbers: 78.20.Bh, 42.79.Dj, 73.20.Mf
\end{abstract}

The observation of extraordinary transmission of a metal film perforated with a two-dimensional periodic array of subwavelength holes [1, 2] has been followed by a considerable amount of theoretical work $3,4,4,5,6,67$. This work has not yet reached the maturity for a full understanding of the precise mechanisms involved in this transmission process. Important global characteristics of the spectra have not yet been addressed satisfactorily: we refer here to the fact that, in comparison with the naive "band structure" of surface plasmons (see equation (2) below), resonances are red shifted and line shapes are asymmetric [3, 4, 5]. In this paper, we will address these issues by developing a unifying model along the lines of the original Fano analysis of the autoionization phenomenon in atomic physics [8] and by experimental confirmation of the essence of the model. We will show that Fano's treatment yields useful insight in the problem of transmission of light through metallic hole arrays by distinguishing two interfering contributions to the transmission process, namely a non-resonant contribution and a resonant one. The latter is associated with surface plasmon (SP) excitations at the interfaces of the hole array. In order to simplify the physics, we allow for resonant SP excitation at one of the interfaces of the hole array only. This assumption is approximately valid for asymmetric arrays, i.e. arrays sandwiched between two different dielectric media (such as air and glass) in which a SP mode can be tuned into resonance on either the one or the other interface, but not on both at the same time. Fabry-Perot mediated coupling between two identical SP's living on separate interfaces [] can induce extra effects in the symmetric case which we will not consider.

The situation studied by Fano is one where the scattering from an input state can take place either directly towards a continuum of states (the scattered states) or via a quasibound state, that is through some type of resonant state, which is then coupled to the scattered states. This process therefore defines two distinct types of scattering channels: one open channel $\psi_{1}$ referring only to the continuum of states and one closed channel $\psi_{2}$ with the resonant state, coupled to the open channel, as symbolized in figure 17. A transition from the input state straight to the open channel $\psi_{1}$ will be called 'direct' or 'non-resonant' as opposed to the other transition path that goes first through the quasibound state of the closed channel $\psi_{2}$ before being scattered and is therefore named 'resonant'. The transition amplitudes associated with each path will interfere to define the total transition prob-
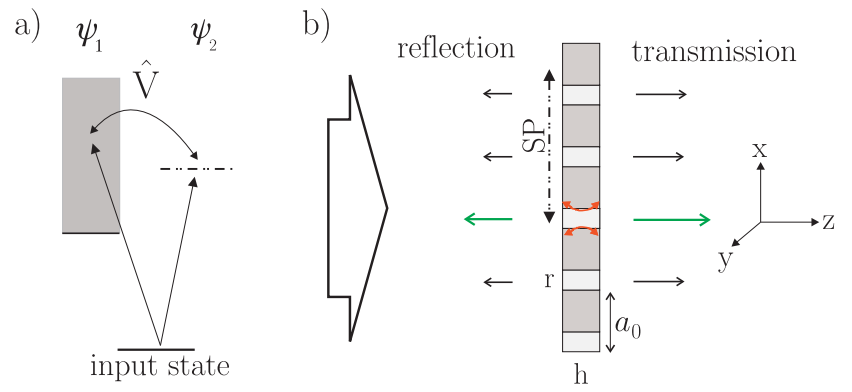

FIG. 1: a) Formal representation of the Fano model for coupled channels. b) Physical picture of the scattering process through the hole array directly (straight arrows) or via SP excitation. The curved arrows inside the hole represent the guided field. The period of the array is denoted $a_{0}$, its thickness $h$ and the radius of each hole $r$.

ability. This situation leads to typical asymmetric resonance line shapes, named (accordingly) as Fano profiles. We have to emphasize that Fano analysis only addresses the global characteristics of the line shape of the spectrum through "structure parameters" but does not give theoretical expressions for these parameters, until a detailed model is defined.

The process of transmission through metallic hole arrays is fundamentally a wave optics problem for which, in principle, a full electromagnetic approach could be given. However, this situation is, at the same time, a scattering problem which can be mapped onto a formal quantum 
Hamiltonian problem, as soon as the various scattering channels are identified. The scattering point of view relates the transition amplitudes that are derived from the Hamiltonian problem to the electromagnetic field scattering amplitudes.

To calculate the total transition probability, one has to solve the coupled channel problem which is defined by the Hamiltonian

$$
\hat{H}=\hat{H}_{1}+\hat{H}_{2}+\hat{V} .
$$

The eigenstates of $\hat{H}_{1}$ correspond to the continuum states of channel $\psi_{1}$ whereas $\hat{H}_{2}$ has one discrete state corresponding to the resonant state in channel $\psi_{2}$. The two channels are coupled via the coupling term $\hat{V}$. Starting from an input state, the derivation of the eigenstates of the full Hamiltonian $\hat{H}$ will give us the transition amplitudes from the input state to the new eigenstates of the coupled channel problem, taking into account the two possible paths.

The first path is non-resonant and simply related to the direct scattering of the field through the subwavelength holes. This contribution will be referred to as Bethe's contribution, based on Bethe's solution to the problem of direct transmission of the electromagnetic field through an infinitely thin ideal metal with subwavelength holes [9]. We will simply adjust this solution to our situation of holes drilled on a real metallic film of finite thickness through a best-fit procedure, thus taking into account in a effective way the influence of these realistic conditions. This non-resonant path will correspond to the $\hat{H}_{1}$ Hamiltonian formally describing a one-dimensional scattering problem, taken along the $z$ direction, as shown in figure [1 Scattering states $\Psi_{E}^{p}$ will be considered as eigenstates of $\hat{H}_{1}$, i.e. $\hat{H}_{1} \Psi_{E}^{p}=E \Psi_{E}^{p}$. They will be defined according to the direction $p$ of the incident plane wave and are given asymptotically far away from the grating. The corresponding scattering amplitudes, related to Bethe's solution, can be gathered into a $S$-matrix $S_{B}$, which is symmetric $\left(S_{B}^{\dagger}=S_{B}\right)$ due to transmission reciprocity property of the array. These states are non-resonant states (defined in the open channel) and belong as free fields to a two-folded continuum: one transmission continuum and one reflection continuum. They are detected as a background in the reflection and transmission spectra. From the quantum point of view, these two continua, specific of an electromagnetic scattering process, are distinguished by the quantum number $p$.

The second path corresponds to a resonant contribution, related to the existence of discrete SP excitations at the illuminated interface of the array. These excitations will be considered as discrete eigenstates $\Psi_{s p}$ of the $\hat{H}_{2}$ hamiltonian, i.e. $\hat{H}_{2} \Psi_{s p}=E_{s p} \Psi_{s p}$. It is well known that these excitations correspond to SP resonances defined on the periodic array by wavevector matching, making use of the array momentum wavevector [10]. At normal inci- dence, such resonances are, to first order, given by complex resonance frequencies

$$
\hat{\omega}_{s p}=\left(n^{2}+m^{2}\right)^{\frac{1}{2}} \frac{2 \pi c}{a_{0}} \sqrt{\frac{\varepsilon_{1}+\varepsilon_{2}}{\varepsilon_{1} \varepsilon_{2}}},
$$

where $(n, m)$ are integers, $a_{0}$ is the period of the array and $\varepsilon_{1}$ and $\varepsilon_{2}$ are respectively the permittivities of the adjacent medium and the metal. The negative imaginary part of $\hat{\omega}_{s p}$ corresponds to the internal (nonradiative) damping of surface plasmon on a smooth interface. This damping represents the coupling between the surface plasmon and metal absorption losses. The real part of $\hat{\omega}_{s p}$ corresponds to the resonance frequencies. In practice, transmission spectra of hole arrays peak at frequencies that are typically $4 \%$ smaller than the value given by the real part of equation (2). This discrepancy, apparent in many experimental works [3, 4, [5], is well known though basically not understood so far.

Due to the array periodicity, the incident field can be converted into a surface plasmon which will eventually be scattered (reflected or transmitted) by reciprocally coupling again with the array. This coupling between the discrete resonance and the scattering states is formally given by the $\hat{V}$ operator (see also figure 1b) which defines the radiative damping of plasmons at the surface of the array, considering the two scattering continua: a radiative transfer directly to free space (to the reflection continuum) and a radiative transfer through the holes (to the transmission continuum). One can give interpretations for these radiative dampings but the key result of this paper relies only on the fundamental idea of the coupling with these two radiative channels. Any interpretation of this coupling, aiming at specifying the mechanisms of the radiative transfers, will not question this result; this is the inherent strength of a Fano-type analysis.

Fano has solved the coupled channel problem giving the normalized eigenstate $\Phi_{E}$ of the full Hamiltonian $\hat{H}$ [8]. The coupling between the resonant state $\Psi_{s p}$ (SP excitation) and the scattering state $\Psi_{E}^{p}$,

$$
\left\langle\Psi_{s p}|\hat{V}| \Psi_{E}^{p}\right\rangle=V_{E}^{p},
$$

is assumed hermitian. It introduces resonance shifts $(\Delta)$ and linewidths $(\Gamma)$ whose expressions can be framed into a Kramers-Kronig relation

$$
\Gamma_{E}=\frac{2 \pi}{\hbar} \sum_{p}\left|V_{E}^{p}\right|^{2} \quad, \quad \Delta_{E}=\frac{1}{2 \pi} \mathcal{P} \int \mathrm{d} E^{\prime} \frac{\Gamma_{E^{\prime}}}{E-E^{\prime}} .
$$

The scattering dynamics of our system are fully contained in the $S$-matrix element corresponding to the global transmission amplitude

$$
t \equiv\left\langle\Phi_{E}|S| i\right\rangle,
$$


with $|i\rangle$ denoting the input state, the left-incident field. This expression takes into account direct scattering from the input state to the continuum of scattering states $\psi_{1}$ (Bethe contribution), specified through the $S$-matrix $S_{B}$, as well as indirect scattering through the resonant state of the closed channel $\psi_{2}$. The expression for the $S$-matrix element (5) takes the shape of a sum of interfering terms from which the expression for the transmission coefficient (transition probability) immediately follows

$$
T=|t|^{2}=\left|t_{B}\right|^{2} \frac{\left[E-\left(E_{s p}+\hbar \Delta\right)+\hbar \delta\right]^{2}}{\left[E-\left(E_{s p}+\hbar \Delta\right)\right]^{2}+[\hbar \Gamma / 2]^{2}},
$$

where $E=\hbar \omega$. The $\delta$ parameter defines the ratio between the resonant transition amplitude and the direct (background) transition amplitude [8]. This expression for the global transmission coefficient reveals the superposition of the non-resonant 'Bethe' contribution, with the transmission coefficient $\left|t_{B}\right|^{2}$, and of the resonant part, with a specific structure that turns out to be exactly a Fano-type profile when written in the natural variables [8]

$$
\epsilon=\frac{E-\left(E_{s p}+\hbar \Delta\right)}{\hbar \Gamma / 2}, q=\frac{2 \delta}{\Gamma} .
$$

The dimensionless $q$ parameter determines the asymmetry of the profile and its sign the corresponding blue $(q>0)$ or red $(q<0)$ tail $[11$.

Our analysis shows that the line shapes are fully determined by 4 structure parameters: the resonance energy $E_{s p}$, identified here as the real part of $\hbar \hat{\omega}_{s p}$, the linewidth $\Gamma$, the resonance shift $\Delta$ and the asymmetry $q$. The linewidth $\Gamma$ includes the plasmon damping terms, i.e. (i) radiative damping, defined by the coupling strength between the surface plasmon discrete state and the scattering states (reflection and transmission continua), and (ii) non-radiative damping defined by the imaginary part of $\hbar \hat{\omega}_{s p}$. The non-radiative damping contribution is generally much weaker than the radiative one, so that the resonance shift $\Delta$, given by the Kramers-Kronig relation (4), is essentially due to the additional radiation damping. This shift can explain the discrepancy between expected resonances (see equation (2) and actual peak positions. It defines therefore non-perturbatively the modified SP's dispersion relation when compared to the SP's dispersion relation on a smooth interface, taking account of the "dressed" character of the SP by coupling to free-field modes. Note that, even if it can easily be parametrized in equations (617), our one-resonance Fano description does not strictly allow for the inclusion of SP-SP scattering since this would involve more than one discrete state. However, this contribution is of higher order in the limit taken here of small holes as compared to the wavelength.

A full electromagnetic theory would deduce this new dispersion relation from the definition of the $S$-matrix of the whole scattering process and from the extraction of its corresponding poles 12,13$]$. The sign of the asymmetry $q$ can be read off directly from the tail of an experimental spectral profile of an isolated SP resonance. Finally, the line shapes of transmission profiles show a characteristic dip at the particular point $\epsilon=-q$ corresponding to a destructive interference effect between the two channels. This destructive interference effect is concomitant to the so called Wood anomaly [14].

The definition of the structure parameters is directly rooted in the introduction of the coupling between a nonresonant and a resonant channel, as essentially shown by the Kramers-Kronig relation (44). This relation provides us with general relations between the damping and the resonance shift. Although we have no explicit expressions for the coupling strength $V_{E}^{p}$ between surface plasmons and scattering states, we can argue generically how it depends on the $(\omega, r, h)$ parameters.

With a simple interpretative model, it is easy to see that the coupling strength is an increasing function of the frequency $\omega$. Towards the reflection continuum, the coupling is directly defined via the holes acting as Herz- or Rayleigh-type dipole emitters induced by the longitudinally polarized surface plasmon. Towards the transmission continuum, the (evanescent) coupling through the holes is considered which is also an increasing function of the frequency. This monotonic behaviour of the coupling strength, that is of the linewidth $\Gamma$ (see equation (4)), implies, when inserted into this Kramers-Kronig relation (4), that the resonance shift $\Delta$ is negative, i.e. a red shift, as noticed by several authors $[3,4,5]$. In fact, the absence of a detailed description for the linewidthresonance shift ratio forces us to consider $\Gamma$ as a free parameter, including both the effects of radiative damping (surface plasmons coupled to scattering states) and non-radiative damping.

This coupling is also strengthened when, at fixed frequency, the hole radius is increased. This lowers the cut-off frequency of the hole waveguide and actually increases the coupling of surface plasmons towards scattering states of the reflection and transmission continua, as the radiating dipoles get stronger. The linewidth is therefore predicted to be an increasing function of the hole radius $\left(\partial_{r} \Gamma>0\right)$ and, at the same time, the red shift of the resonance should be more pronounced $\left(\partial_{r}|\Delta|>0\right)$. These variations have been observed experimentally [4].

If the thickness $h$ of the array is increased, the coupling towards the transmission continuum decreases. In the hypothetic limit of infinite thickness, only radiation damping on the reflection interface remains. As $h$ is increased, the progressive disappearance of the radiative channel through the holes, due to higher (non-radiative) losses inside the waveguide, induces a narrowing of the linewidth $\left(\partial_{h} \Gamma<0\right)$ and consequently a reduced red shift $\left(\partial_{h}|\Delta|<0\right)$ of resonance. Two regimes of variation for linewidths and resonance shifts can be understood from the introduction of a typical thickness above which the 
radiative coupling is mainly towards the reflection continuum. The variations $\partial_{h} \Gamma<0$ and $\partial_{h}|\Delta|<0$, along with the existence of a critical thickness, are again in agreement with recent measurements 15].

Figure 2 shows measurement transmission data from one of our experiments, for the $(1,0) \mathrm{SP}$ resonance on the air-metal side of our array. This array is made of a 200

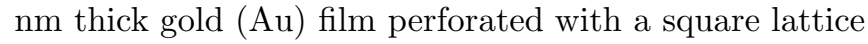
of $70 \mathrm{~nm}$ radius holes spaced with a $700 \mathrm{~nm}$ lattice period. Our sample is asymmetric: the Au film is evaporated on a glass substrate with a $10 \mathrm{~nm}$ thick bonding layer of titanium (Ti). Strong absorption in this bonding layer prevents any surface plasmon from being excited on the metal-glass interface and, as such, guarantees that only one interface contributes, in agreement with the general frame of our model. This profile has a wavelength red tail, i.e. $q$ is negative [11]. We notice that the position of the measured peak (at $\lambda=739 \mathrm{~nm}$ ) is $\sim 4 \%$ red shifted when compared to the position of the corresponding SP resonance $\lambda_{s p}=712 \mathrm{~nm}$ naively expected from equation (2) with a period of the array $a_{0}=700 \mathrm{~nm}$.

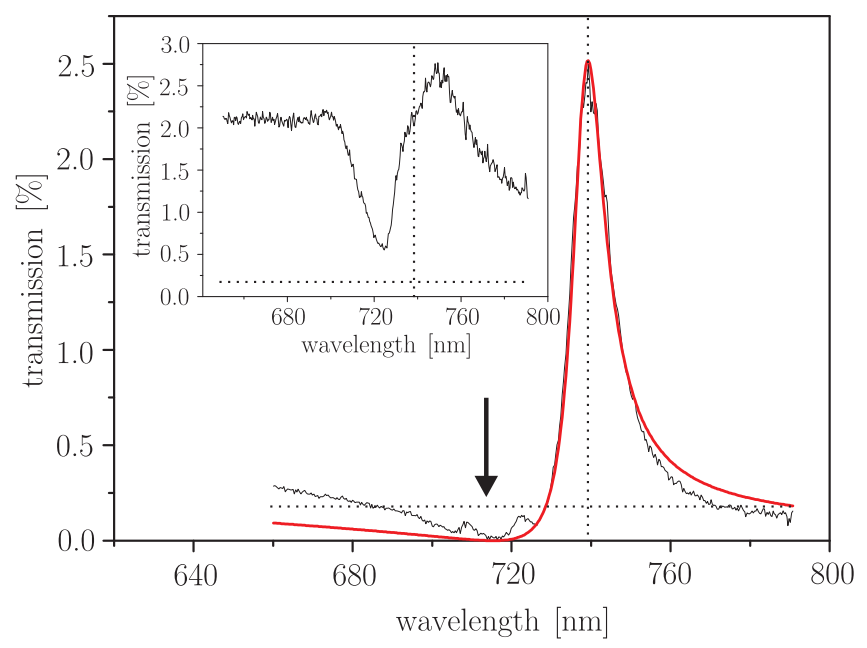

FIG. 2: Experimental transmission spectrum of the air-metal $(1,0) \mathrm{SP}$ resonance, obtained with an Au film of thickness $200 \mathrm{~nm}$ with $a_{0}=700 \mathrm{~nm}$ and $r=70 \mathrm{~nm}$. The smooth curve shows the fitted Fano profile (6) by adjusting freely the natural variables of (7). The vertical dotted line marks the position of the peak whereas the arrow shows the position of the resonance given by equation (2). The horizontal dotted line shows the Fano background level. Inset: Experimental transmission spectrum for the same array, but now in a film only $100 \mathrm{~nm}$ thick. The former dotted "cross" has been superimposed, emphasizing the background increase and the dispersive line shape.

We attribute this shift to a negative $\Delta$ parameter, as to be expected from the above general arguments. It should be noticed that the various parameters of equation (6) are intertwined in their effects on the line shape. Shifts of SP resonances are determined for one part by $\Delta$ and for the other part by the asymmetry itself. In natural units, the peak of the Fano profile is centered on $\epsilon=1 / q$. A red tail asymmetry $(q<0)$ induces therefore an extra red shift, though small (less than 1\%) when compared to the red shift that is expected from $\Delta$. On the other hand, the non-resonant transmission coefficient scales as $\left|t_{B}\right|^{2} \sim(r / \lambda)^{4}$, according to Bethe's theory [9]. This defines a non-resonant background colored towards the blue. The Fano peak will therefore be blue-shifted when superposed on this background. This blue shift can again be neglected when compared to the red shift $\Delta$.

In figure 2 we have superposed a fitting of the experimental curve with a profile defined by equation (6) [16]. The fitted parameters indicate a red shift of $4 \%$ determined by $\Delta<0$ and a relative linewidth of about $3 \%$, that is of the order of the shift. The background is found to be only about $0.2 \%$, which is consistent with Bethe's calculations for this system. Below $710 \mathrm{~nm}$, the tail of the air-metal $(1,1)$ resonance is emerging.

As mentioned above, the value of the Fano asymmetry $q$ characterizes the ratio between the resonant transition amplitude and the background transition amplitude. Our experimental curve confirms this scaling with an asymmetry of the order of 4 which can also be deduced from the fitting parameters. As a check of the Fano description, we have reduced the Au film thickness from $200 \mathrm{~nm}$ to $100 \mathrm{~nm}$ with the same $10 \mathrm{~nm}$ thick Ti layer. With this reduction, the film thickness is only a few times the $\mathrm{Au}$ skin depth allowing another direct leakage channel. This should increase the non-resonant background contribution and thus lower the value of $q$. We have confirmed this experimentally: see the inset of figure 2 where the background level is increased and the profile of the resonance gets close to a dispersive shape which corresponds to a lower $q$ value.

In conclusion, we have shown that a Fano analysis is a unifying tool to understand hole array spectra since simple scaling laws can be inferred. Only one resonance has been addressed in this paper and one should now extend this work to the collection of SP excitations on the array, i.e. to multichannel theory.

Note added in proof - During the process of publication of this paper, we learned about the theoretical work of Sarrazin el al. [17] which shows important overlapping with our. Nevertheless, we put here the emphasis on the concept of coupled scattering channels in the context of experimental spectra, in order to understand corresponding lineshapes.

Acknowledgments - This work forms part of the program of FOM; we would like to thank G. Nienhuis for useful discussions.

[1] T.W. Ebbesen et al., Nature 391, 667 (1998) 
[2] E. Altewischer et al., Nature 418, 304 (2002)

[3] H.F. Ghaemi et al., Phys. Rev. B 58, 6779 (1998)

[4] L. Salomon et al., Phys. Rev. Lett. 86, 1110 (2001)

[5] A. Krishnan et al., Opt. Comm. 200, 1 (2001)

[6] E. Popov et al., Phys. Rev. B 62, 16100 (2000)

[7] L. Martín-Moreno et al., Phys. Rev. Lett. 86, 1114 (2001)

[8] U. Fano, Phys. Rev. 124, 1866 (1961)

[9] H.A. Bethe, Phys. Rev. 66, 163 (1944)

[10] H. Raether, Surface Plasmons on Smooth and Rough Surfaces and on Gratings (Springer-Verlag, 1988)

[11] The transmission coefficient is measured as a function of the wavelength. A negative asymmetry $q_{E}<0$ for frequency spectra immediately corresponds to a positive asymmetry $q_{\lambda}>0$ for wavelength spectra.

[12] J.B. Pendry, and A. MacKinnon, Phys. Rev. Lett. 69, 2772 (1992)

[13] W.L. Barnes et al., Phys. Rev. B 51, 11164 (1995)

[14] A. Hessel, and A.A. Oliner, App. Opt. 4, 1275 (1965)

[15] A. Degiron et al., App. Phys. Lett. 81, 4327 (2002)

[16] The two wiggles seen around the dip of the profile are an experimental artefact due to non perfect alignment of the incident beam and the normal axis of the array.

[17] M.Sarrazin, J.-P. Vigneron, and J.-M. Vigoureux, Phys. Rev. B 67, 085415 (2003) 